\title{
A Novel Approach to Evaluate Reduced Inter Symbol Interference in UFMC Systems
}

\author{
Elavel Visuvanathan. $\mathrm{G}^{\mathrm{a}, 1}$ and Jaya. $\mathrm{T}^{\mathrm{b}}$ \\ ${ }^{\mathrm{a}, 1}$ Research Scholar, Dept. of ECE, Vels Institute of science, Technology and Advanced \\ Studies (VISTAS) \\ ${ }^{\mathrm{b}}$ Assistant Professor, Dept. of ECE, Vels Institute of science, Technology and Advanced \\ Studies (VISTAS)
}

\begin{abstract}
The UFMC modulation scheme has been proposed as a solid competitive framework for future portable fifth generation communication. UFMC can be considered as a candidate waveform for $5 \mathrm{G}$ communications since it gives strength against Inter Symbol Interference (ISI) [1]. Inter-symbol interference prompted error can make the receiver neglect to reproduce the original data. Equalizers in the receivers, which are extraordinary sorts of filters, moderate the direct twisting created by the channel [2]. On the off chance that the channel's time-fluctuating qualities are known from the earlier, at that point, the ideal setting for equalizers can be worked out. But in practical systems the channel's timechanging attributes are not known from the earlier, so adaptive equalization method is applied in this paper based on the LMS algorithms. Adaptive equalizers are adjusted, or change the estimation of its taps as time advances [3].
\end{abstract}

Keywords. UFMC, Inter Symbol Interference, adaptive equalization, LMS

\section{Introduction}

The appetite of human culture for more data transmission based applications constraining the cellular industry to progress in the direction of better advancements and is fuelling the improvement of fifth-generation research. By 2020 edge fifthgeneration cellular access will be a reality and as of now, trails are going on over the world. At present OFDM method is broadly utilized in remote communications just as in numerous advanced communications for its effectiveness. Notwithstanding, it has disadvantages high. In OFDM Cyclic Prefix causes ISI which is caused because of postponement in the conveyance of the channel is higher than CP length.

${ }^{1}$ G. Elavel Visuvanathan, Research Scholar, Dept. of ECE, Vels Institute of science, Technology and Advanced Studies (VISTAS)

E-mail: elavel.visuvanathan@gmail.com 


\section{Universal Filter Multi Carrier}

\subsection{UFMC Transmitter}

For the most part in filtered orthogonal frequency-division multiplexing, the whole for band is filtered while in Filter Bank Multicarrier singular subcarriers are filtered. Be that as it may, in the UFMC gathering of subcarriers are filtered. UFMC uses QAM to maintain magnificent proportion, which works with existing MIMOs. The entire area of the UFMC transmitter is shown in Figure 1.

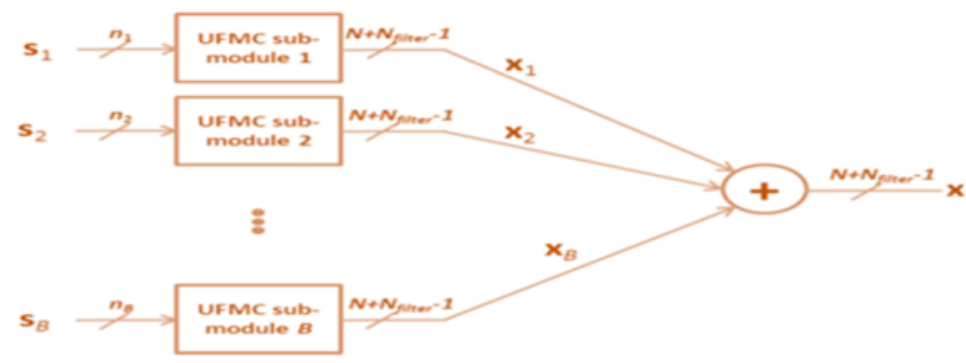

Figure 1. Basic UFMC transmitter structure

Here the entire band of sub-carriers "N" is separated into a few sub-bands. Every sub-band has a static quantity of sub-carriers. Not all sub-bands in the transmission segment have to be used for the transmission. The IFFT is used to eliminate the impediment of the sub-band carrier.

\subsection{Material and Methods}

With the unimaginable improvement of Internet advancements, productive fast data transmission methods over communication channels have become a need of the day. The data transmitted through a band-restricted communication channel experiences direct, nonlinear and added substance bends. So as to decrease the impacts of these contortions an equalizer is utilized at the receiver end.

Figure 2 shows a block-diagram of a communication system with an adaptive equalizer in the receiver. In the event that $\mathrm{f}(\mathrm{t})$ is the consolidated complex baseband drive reaction of the transmitter, and $b(t)$ is the original data signal, channel and the $\mathrm{RF} / \mathrm{IF}$ areas of the receiver, the signal got by the equalizer might be communicated as

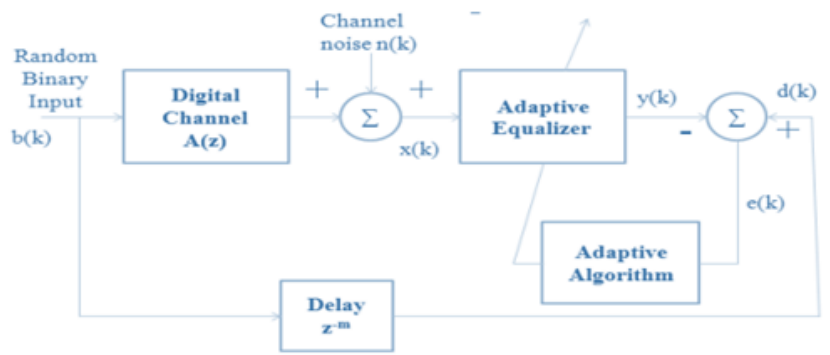

Figure 2. Block Diagram of Adaptive Equalization 


$$
x(t)=f *(t) \otimes b(t)+m_{2}(t)
$$

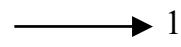

Where $n b(t)$ is the base-band noise at the contribution of the equalizer, $f^{*}(t)$ is the difficult conjugate of $\mathrm{f}(\mathrm{t})$ and 0 indicates the convolution activity.

$$
y(t)=n_{\mathrm{b}}(t) \otimes h_{\varepsilon q}(t)+f^{\prime} *(t) \otimes b(t) \otimes h_{q q}(t) \longrightarrow 2
$$

The complex base-band motivation reaction of AFE is given by

$$
h_{\mathrm{rqg}}(\mathrm{t})-\Sigma_{\mathrm{n}} \mathrm{C}_{\mathrm{n}} \delta(\mathrm{t} \mathrm{t}-\mathrm{nT})
$$

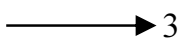

Here $g(t)$ is the common motivational response of the transmitter, the original source data.

$$
g(t)=f *(t) \otimes h_{\mathrm{eq}}(t)=8(t)
$$

The objective of equalization is to fulfil condition (4). In the recurrence space, condition (2.4) can be communicated as

$$
\text { Heq (f) } F^{*}(-f)=1
$$

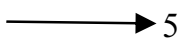

\section{Result}

The simulation of the model under examination was completed utilizing the MATLAB. In figure 3, the performance of the proposed filters is introduced, from which we can obviously observe their trade-offs gave by the differing point. From the figure 3 attenuation and pass-band ripple it clearly shows that value of trade-off starts from minimum value and slowly reaches maximum values.
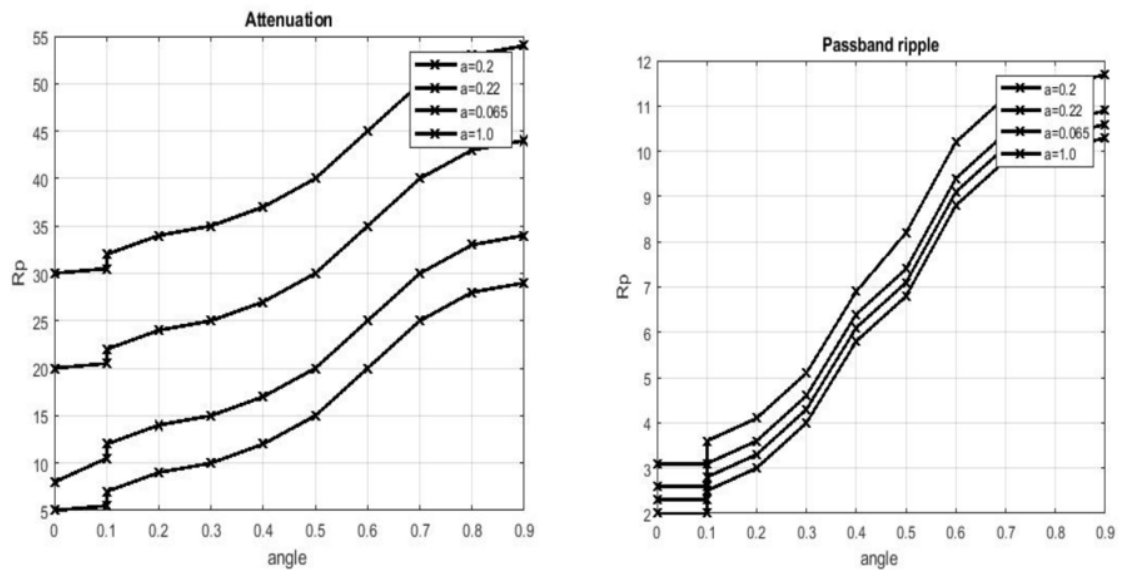

Figure 3. Attenuation and Pass-band ripple 
Table 1. performance measure of Dolph-Chebyshev Filter and Adaptive Equalization Filter

\begin{tabular}{cccccccc}
\hline \multicolumn{3}{c}{ Dolph-Chebyshev Filter } & \multicolumn{3}{c}{ Adaptive Equalization Filter } \\
\hline PARAMETERS & $\begin{array}{c}\text { SNR } \\
\text { (in } \\
\text { dB) }\end{array}$ & BER & Data Rate & PARAMETERS & $\begin{array}{c}\text { SNR } \\
\text { (in } \\
\text { dB) }\end{array}$ & BER & $\begin{array}{c}\text { Data } \\
\text { Rate }\end{array}$ \\
\hline ANGLE $=0$ & 11 & 0.125 & 2 & ANGLE $=0$ & 12 & 0.0165 & 2 \\
ANGLE $=20$ & 12 & 0.124 & 4 & ANGLE $=20$ & 13 & 0.0154 & 4 \\
ANGLE $=60$ & 14 & 0.459 & 8 & ANGLE $=60$ & 13 & 0.0147 & 8 \\
ANGLE $=180$ & 10 & 0.668 & 16 & ANGLE $=180$ & 13 & 0.0258 & 16 \\
ANGLE $=270$ & 11 & 0.998 & 32 & ANGLE $=270$ & 15 & 0.0965 & 32 \\
ANGLE $=360$ & 11 & 0.852 & 64 & ANGLE $=360$ & 15 & 0.0258 & 64 \\
\hline
\end{tabular}

In Figure 4 shows the comparative plot of existing wave shaping filter, adaptive equalization and without equalization.
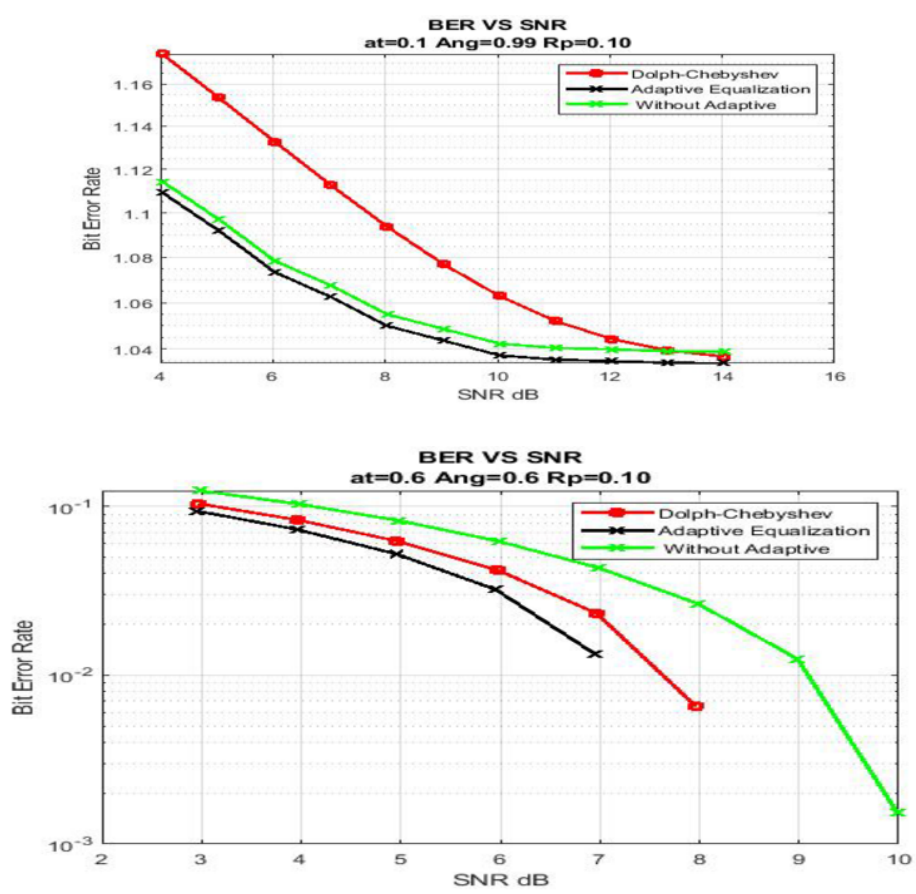


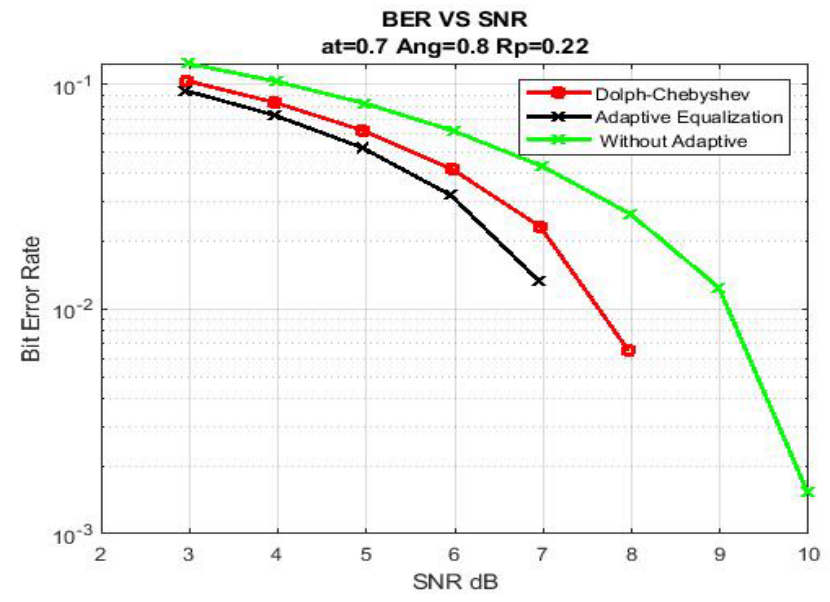

Figure 4.Comparative plot of existing Wave Shaping Filter.

\section{Conclusion}

The performance was analyzed by varying the filter lengths, the type of filters and the Bit Error Rate and the results have been observed. It has been seen that there would be a decrease in the side flap levels with an expansion in the filter length along these lines lessening the out-of-band transmission.

\section{Reference}

[1] Li, Ye Geoffrey, and Gordon L. Stuber, eds. orthogonal frequency division multiplexing for wireless communications. Springer Science \& Business Media, 2006.

[2] Zhang, Haijian.H, Didier Le Ruyet, Daniel Roviras, Yahia Medjahdi, and Hong Sun. Spectral efficiency comparison of OFDM/FBMC for uplink cognitive radio networks. EURASIP Journal on Advances in Signal Processing 2010 (2010): 4.

[3] Visuvanathan Ganesan E, Thangappan J. CFO and STO estimation and correction in multicarrier communications using linear filter bank multicarrier. Trans Emerging Tel Tech. 2021;e4229.

[4] Bochechka.G .Methods of channel estimation based on built in pilot-signals in OFDM systems. Telecommunications and Transport, 2009, No3, pp. 38-42.

[5] F.Hu, Opportunities in 5G Networks a research and development perspective, CRC Press, 2016

[6] Tullberg, Hugo, Heinz Droste, Mikael Fallgren, Peter Fertl, David Gozalvez-Serrano, Eiman Mohyeldin, Olav Queseth, and Yngve Seien. METIS research and standardization: A path towards a 5G system. In Globecom Workshops (GC Wkshps), 2014, pp. 577-582. IEEE, 2014.

[7] Wunder, Gerhard, Peter Jung, Martin Kasparick, Thorsten Wild, Frank Schaich, Yejian Chen, Stephan Ten Brink et al. 5GNOW: nonorthogonal, asynchronous waveforms for future mobile applications. IEEE Communications Magazine 52, no. 2 (2014): 97-105. 